\title{
Spin polarization of electrons by ultraintense lasers
}

\author{
D. Del Sorbo, ${ }^{1}$ D. Seipt,${ }^{2,3}$ T. G. Blackburn, ${ }^{4}$ A. G. R. Thomas, ${ }^{3,2}$ C. D. Murphy, ${ }^{1}$ J. G. Kirk, ${ }^{5}$ and C. P. Ridgers ${ }^{1}$ \\ ${ }^{1}$ York Plasma Institute, Department of Physics, University of York, York YO10 5DD, United Kingdom \\ ${ }^{2}$ Cockcroft Institute, Daresbury Laboratory, Warrington WA4 4AD, United Kingdom \\ ${ }^{3}$ Department of Physics, Lancaster University, Lancaster LA1 4YB, United Kingdom \\ ${ }^{4}$ Department of Physics, Chalmers University of Technology, Gothenburg SE-41296, Sweden \\ ${ }^{5}$ Max-Planck-Institut für Kernphysik, Saupfercheckweg 1, Heidelberg D-69117, Germany
}

(Received 30 January 2017; published 11 October 2017)

\begin{abstract}
Electrons in plasmas produced by next-generation ultraintense lasers $\left(I>5 \times 10^{22} \mathrm{~W} / \mathrm{cm}^{2}\right)$ can be spin polarized to a high degree $(10 \%-70 \%)$ by the laser pulses on a femtosecond time scale. This is due to electrons undergoing spin-flip transitions as they radiate $\gamma$-ray photons, preferentially spin polarizing in one direction. Spin polarization can modify the radiation reaction force on the electrons, which differs by up to $30 \%$ for opposite spin polarizations. Consequently, the polarization of the radiated $\gamma$-ray photons is also modified: the relative power radiated in the $\sigma$ and $\pi$ components increases and decreases by up to $30 \%$, respectively, potentially reducing the rate of pair production in the plasma by up to $30 \%$.
\end{abstract}

DOI: 10.1103/PhysRevA.96.043407

\section{INTRODUCTION}

At the intensities which will be reached by next-generation ultraintense lasers $\left(\gtrsim 5 \times 10^{22}-10^{24} \mathrm{~W} / \mathrm{cm}^{2}\right)$, such as several of those comprising the Extreme Light Infrastructure [1], light-matter interactions are predicted to reach the new quantum electrodynamic (QED) plasma regime. Matter in the laser focus is rapidly ionized, creating a plasma whose behavior is characterized by the interplay of relativistic plasma and "strong-field" quantum electrodynamic processes [2]. Understanding this interplay is of fundamental interest: this regime is similar to that inferred to exist in extreme astrophysical environments such as pulsar [3] and active black-hole magnetospheres [4]. The QED processes can strongly modify the plasma's behavior, for example, leading to complete absorption of the laser pulse, with consequences for potential applications of these lasers, ranging from compact particle accelerators [5] to X-ray source generation [2]. Despite its importance, the role of fermion spin in collective high-intensity laser-matter dynamics has rarely been considered [6].

The important strong-field QED processes in laser-created QED plasmas are [2,7,8] (i) incoherent emission of $\mathrm{MeV}$ energy $\gamma$-ray photons by electrons and positrons on acceleration by the macroscopic electromagnetic fields in the laserproduced plasma (strongly nonlinear Compton scattering), with the resulting radiation reaction strongly modifying the dynamics of the emitting electron or positron $[9,10]$, and (ii) pair creation by the emitted $\gamma$-ray photons in the same electromagnetic fields (the multiphoton Breit-Wheeler process [11]). For example, nonlinear Compton scattering and the resulting radiation can lead to almost complete laser absorption $[12,13]$; pair cascades (where pairs emit further $\gamma$-ray photons, which generate even more pairs), can lead to the creation of critical density pair plasmas [14-17]. It is therefore essential

Published by the American Physical Society under the terms of the Creative Commons Attribution 4.0 International license. Further distribution of this work must maintain attribution to the author(s) and the published article's title, journal citation, and DOI. that we correctly include these QED processes in our models of the interaction of next-generation laser pulses with matter. Previous treatments of $\gamma$-ray photon emission in QED plasmas have averaged over the spin.

In this article we demonstrate a process, analogous to the Sokolov-Ternov effect in a strong magnetic field [18,19], in which the electrons in laser-generated QED plasmas rapidly spin polarize due to asymmetry in the rate of spin-flip transitions, i.e., interactions where the spin changes sign during the emission of a $\gamma$-ray photon. We discuss several signatures of the spin polarization of the plasma. (i) Consideration of the spin of the electrons leads to a quantum correction to the radiation reaction force: in the energetically favorable spin configuration the total power radiated and so radiation reaction force are enhanced compared to the unfavorable configuration, leading to an enhancement in total power radiated by the plasma compared with the prediction assuming the spin is unpolarized. (ii) The relative energy emitted in the two possible polarizations of the $\gamma$-ray photons is modified. (iii) This modification could decrease the rate of pair production.

Spin-polarized electron beams are important in highenergy physics; the use of properly polarized beams in electron-positron colliders can suppress the standard-model background in searches for new physics beyond the standard model [20]. Spin-polarized beams are also used in electron spectroscopy for studying surface and thin-film magnetism and the electronic structure of metal semiconductor surfaces and films $[21,22]$. Spin polarizing plasma may enable applications of ultraintense lasers in these areas.

This paper is organized as follows. In Sec. II we demonstrate the laser-induced process of electron spin polarization by deriving a simple predictive model and applying it to the case of electrons orbiting at the magnetic node in the field of two counterpropagating lasers. In Sec. III we discuss signatures and consequences of spin polarization. Finally, in Sec. IV we draw conclusions.

\section{SPIN POLARIZATION BY LASER PULSES}

We focus on the case of electrons orbiting in a rotating electric field, a configuration that may be realized in the 
plasma created at the magnetic node of two colliding, circularly polarized laser pulses. The electric field $\boldsymbol{E}$ at the magnetic node, say, the plane $z=0$, rotates with constant amplitude [23]. Consequently, electrons subjected to $\boldsymbol{E}$ also rotate in the plane $z=0$ with (normalized) velocity $\boldsymbol{\beta}$.

To describe the spin-polarization dynamics of electrons in the rotating electric field (indeed, in any arbitrary electromagnetic field) we need to use a proper nonprecessing spin-polarization basis $\zeta$ for which $d \zeta / d \tau=0$ [24], defined in the rest frame of the electron, where $\tau=t / \gamma$ is the proper time. Spin-up and spin-down electron states, defined with respect to that basis, do not mix. According to the Bargmann-MichelTelegdi equation [25], which describes the precession of the expectation value of the spin-polarization vector in an external electromagnetic field [26], the only nonprecessing spin basis for an electron rotating in the rotating electric field is

$$
\zeta=\frac{E \times \boldsymbol{\beta}}{\|\boldsymbol{E} \times \boldsymbol{\beta}\|}=\boldsymbol{e}_{z} .
$$

We will therefore consider spin polarization in the transverse direction where $\zeta$ is perpendicular to $\beta$. $\zeta$ is analogous to the direction along the magnetic field in the discussion of the Sokolov-Ternov effect.

Each electron in the rotating electric field has a projection of its spin in the direction of the vector $\zeta$ of $s \hbar / 2$, where $s= \pm 1$ and $\hbar$ is Planck's constant. We therefore divide the plasma electron population into two fractions $n^{s}$, characterized by their spin projection being parallel ( $s=1=\uparrow$, higher energy) or antiparallel ( $s=-1=\downarrow$, lower energy) to $\zeta$. The number density of electrons with spin $s=\downarrow$, for instance, evolves according to the master equation

$$
\frac{d}{d \tau} n^{\downarrow}(t)=\frac{d N^{\uparrow \downarrow}}{d \tau} n^{\uparrow}(t)-\frac{d N^{\downarrow \uparrow}}{d \tau} n^{\downarrow}(t) .
$$

The $\gamma$-ray photon emission rates $d N^{s s^{\prime}} / d \tau=\sum_{\rho} d N_{\rho}^{s s^{\prime}} / d \tau$, summed over photon polarization states $\rho$, describe the transitions from one spin state to the other on emission of a $\gamma$-ray photon, i.e., spin-flip transitions. An analogous equation holds for $n^{\uparrow}$, the number density of electrons with spin $s=\uparrow$.

\section{A. Rate of spin-flip transitions}

The expressions for the rate of spin-flip transitions can be simplified when the strength parameter $a_{0}=$ $85.5 \lambda / \mu \mathrm{m} \sqrt{I /\left(10^{22} \mathrm{~W} / \mathrm{cm}^{2}\right)}$ of the laser electromagnetic waves is large. In that case, the formation phase interval for the emission of a photon becomes very short, $\propto 1 / a_{0}$, and multiple-photon emissions can be considered incoherent. $a_{0}$ is indeed large in the cases considered here, where the intensity of each of the counterpropagating laser pulses $I$ is $\gg 10^{18} \mathrm{~W} / \mathrm{cm}^{2}$ and $\lambda=1 \mu \mathrm{m}$, a typical wavelength of ultraintense lasers that is assumed in all our calculations. It is also the case that the electric field at the magnetic node is much less than the critical field of QED: the Sauter-Schwinger field $E_{S}=m_{e}^{2} c^{3} /(e \hbar)$ [27], where $m_{e}$ and $e$ are the electron mass and charge, respectively, and $c$ is the speed of light (all in Gaussian units). Under these two assumptions the rates of $\gamma$-ray photon emission (and pair production) are well described by the corresponding rates in constant crossed electric and magnetic fields $[8,18,28,29]$. The rates of photon emission in this constant crossed-field approximation depend only on the electron's initial and final spin projections $s$ and $s^{\prime}$, the emitted photon's polarization $\rho$, and the quantum efficiency parameter $\eta=E_{R F} / E_{S}$ [28]. Here, $E_{R F}=\gamma\|\boldsymbol{E}\|$ is the electric field's magnitude in the electron's instantaneous rest frame. The electron-spin- and photon-polarization-dependent photon emission rates read $[18,30]$

$$
\frac{d N_{\rho}^{s s^{\prime}}}{d \tau}=\mathcal{P}_{\text {class }} \int_{0}^{\eta / 2} d \chi \frac{d y}{d \chi} \frac{F\left(\eta, \chi, s, s^{\prime}, \rho\right)}{\hbar \omega / \gamma},
$$

with the classical dipole radiation emission power

$$
\mathcal{P}_{\text {class }}=\frac{2 m_{e}^{2} c^{3} e^{2} \eta^{2}}{3 \hbar^{2}} .
$$

The photon quantum parameter

$$
\chi=\frac{\hbar \omega E}{2 m_{e} c^{2} E_{S}}
$$

depends on the $\gamma$-ray photon energy

$$
\hbar \omega=\frac{\gamma m_{e} c^{2} \xi y}{1+\xi y}
$$

with $\xi=3 \eta / 2$ and

$$
y=\frac{4 \chi}{3 \eta(\eta-2 \chi)} .
$$

$F\left(\eta, \chi, s, s^{\prime}, \rho\right)$ is the spin- and photon-polarization-dependent quantum synchrotron function. It is given by

$$
\begin{aligned}
F\left(\eta, \chi, s, s^{\prime}, \rho\right)= & \frac{9 \sqrt{3} y}{16 \pi(1+\xi y)^{4}} \\
& \times\left(\frac { 1 + s s ^ { \prime } } { 2 } \left\{( 1 + \frac { 1 } { 2 } \xi y ) ^ { 2 } \left[\int_{y}^{\infty} K_{5 / 3}(x) d x\right.\right.\right. \\
& \left.+\rho K_{2 / 3}(y)\right]+\left[\frac{\xi^{2} y^{2}}{2} \int_{y}^{\infty} K_{1 / 3}(x) d x\right. \\
& \left.\left.-s^{\prime}(2+\xi y) \xi y K_{1 / 3}(y)\right] \frac{1+\rho}{2}\right\} \\
& +\frac{1-s s^{\prime}}{2} \frac{\xi^{2} y^{2}}{4}\left\{\int_{y}^{\infty} K_{5 / 3}(x) d x-\rho K_{2 / 3}(y)\right. \\
& \left.\left.+\left[2 \int_{y}^{\infty} K_{1 / 3}(x) d x-4 s^{\prime} K_{1 / 3}(y)\right] \frac{1-\rho}{2}\right\}\right)
\end{aligned}
$$

where $K$ is the modified Bessel function of the second kind. The variable $\rho$ refers to the photon polarization, where $\rho=$ $1=\sigma$ (polarized orthogonal to $\zeta$ ) and $\rho=-1=\pi$ (polarized in the direction of $\zeta$ ).

Figure 1(a) shows the rates in Eq. (3), summed over photon polarizations, $d N^{s s^{\prime}} / d \tau=d N_{\sigma}^{s s^{\prime}} / d \tau+d N_{\pi}^{s s^{\prime}} / d \tau$, as functions of laser power of one of the counterpropagating pulses (the rates depend on $\eta$, which in turn depends on $a_{0}$, as explained in the next paragraph) and normalized to the unpolarized rate for a single electron $d N / d \tau=\sum_{s s^{\prime}} d N^{s s^{\prime}} / d \tau{ }^{1}$ The

\footnotetext{
${ }^{1}$ Our $d N / d \tau$ is twice that defined in Refs. [9,29] because two electron populations, characterized by their polarization, are involved.
} 


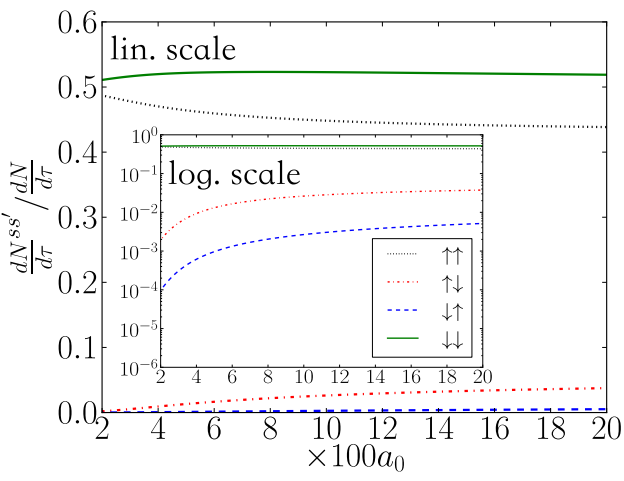

(a)

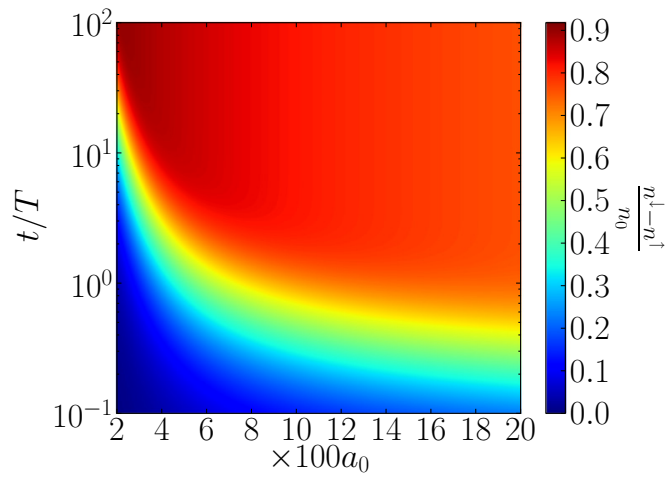

(b)

FIG. 1. (a) The rates in Eq. (3), summed over photon polarization and normalized to the unpolarized rate $d N / d \tau$, as functions of the strength parameter of the laser electromagnetic waves $a_{0}$. (b) Degree of electron spin polarization antiparallel as a function of $a_{0}$ and of time normalized to the laser period $T \approx 3.33$ fs.

rate of processes resulting in a final spin aligned antiparallel to $\zeta\left(d N^{\downarrow \downarrow} / d \tau+d N^{\uparrow \downarrow} / d \tau\right)$ is higher than those resulting in a final spin aligned parallel $\left(d N^{\uparrow \uparrow} / d \tau+d N^{\downarrow \uparrow} / d \tau\right)$ due to the difference in the rates of spin flip. This means that the electron spin tends to align itself antiparallel to $\zeta$ on a time scale which we denote as the spin-polarization time $t_{\mathrm{pol}}$.

In order to calculate the rates in Fig. 1(a) we had to determine $\eta$. The electrons rotating in the electric field reach a steady state when the radiative losses due to $\gamma$-ray photon emission balance the acceleration due to the electric field. In this case [12] $\eta \approx 206 \varepsilon_{\text {rad }} \gamma^{2}$, where

$$
\varepsilon_{\mathrm{rad}}=\frac{4 \pi e^{2}}{3 m_{e} c^{2} \lambda} \approx 1.18 \times 10^{-8}
$$

and the electron's Lorentz factor is given by

$$
\left[g(\eta) \varepsilon_{\mathrm{rad}} \gamma^{4}\right]^{2}+\gamma^{2}=a_{0}^{2} .
$$

The factor

$$
g(\eta)=\sum_{s s^{\prime} \rho} \frac{\mathcal{P}_{\rho}^{s s^{\prime}}}{\mathcal{P}_{\text {class }}}=\sum_{s s^{\prime} \rho} \int_{0}^{\eta / 2} d \chi \frac{d y}{d \chi} F\left(\eta, \chi, s, s^{\prime}, \rho\right)
$$

accounts for the reduction of the power radiated due to quantum effects [9], compared to classical emission, where $\mathcal{P}^{s s^{\prime}}$ is the power radiated during an emission where the initial and final spins are $s$ and $s^{\prime}$, respectively.

The Sokolov-Ternov effect in a magnetic field has been observed after $1 \mathrm{~h}$ in storage rings [18], for which the quantum efficiency parameter $\eta \sim 10^{-6}$ or less [31]. Next-generation ultraintense lasers are expected to reach more extreme regimes, in which $\eta \sim 0.1-1$ [23]. These high values of $\eta$ can also be achieved in the interaction of relativistic particles with strong crystalline fields [32], as has been experimentally observed [33].

\section{B. Spin-polarization time}

We may determine the degree of spin polarization antiparallel to $\mathbf{E} \times \beta$ (i.e., $\downarrow$ ) in time $t$ by solving (2) with the rates (3). We find that it evolves as

$$
\frac{n^{\downarrow}-n^{\uparrow}}{n_{0}}=\frac{\frac{d N^{\uparrow \downarrow}}{d t}-\frac{d N^{\downarrow \uparrow}}{d t}}{\frac{d N^{\uparrow \downarrow}}{d t}+\frac{d N^{\downarrow \uparrow}}{d t}}\left(1-e^{-t / t_{\mathrm{pol}}}\right),
$$

with the spin-polarization time $t_{\text {pol }}=\gamma\left(d N^{\uparrow \downarrow} / d \tau+\right.$ $\left.d N^{\downarrow \uparrow} / d \tau\right)^{-1}$ and assuming initially unpolarized electrons with total density $n_{0}=n^{\uparrow}+n^{\downarrow}$. By inverting the signs of $s$ and $s^{\prime}$ this equation describes the polarization of positrons as well [34].

In Fig. 1(b) we plot the solutions of Eq. (12) as a function of time, normalized to the laser period $T=\lambda / c \approx 3.33 \mathrm{fs}$, and of $a_{0}$. We see that for laser intensities just beyond the current limit $\left(a_{0} \approx 200 ; I \approx 5 \times 10^{22} \mathrm{~W} / \mathrm{cm}^{2}\right)$ a significant $(\approx 10 \%)$ spin polarization is expected to occur rapidly, i.e., within a single laser period. Note that $\gtrsim 50 \%$ spin polarization is expected in one laser period for lasers of intensity well within the reach of next-generation laser systems $\left(a_{0} \gtrsim 600\right.$; $I \gtrsim 5 \times 10^{23} \mathrm{~W} / \mathrm{cm}^{2}$ ), and a maximum spin polarization of $70 \%$ after one laser period is expected at a laser intensity of $5 \times 10^{24} \mathrm{~W} / \mathrm{cm}^{2}\left(a_{0} \approx 2000\right)$.

The orbit considered here is unstable on a time scale of the order of the laser period [35]. However, as we have shown, a high degree of spin polarization can, indeed, occur on such a short time scale.

\section{CONSEQUENCES OF SPIN POLARIZATION}

Let us now discuss several immediate consequences of the electron polarization on the subsequent QED plasma dynamics. Figure 2(a) shows the spin-dependent $g^{s}(\eta)=$ $\sum_{s^{\prime} \rho} \mathcal{P}_{\rho}^{s s^{\prime}} / \mathcal{P}_{\text {class }}$ by polarized electrons. The intensity of emission from an electron initially in the state $s=\downarrow$ is up to $30 \%$ higher than that from an electron initially in the state $s=\uparrow$. Hence, the power radiated and, consequently, the radiation reaction force depend on the spin of the electron. This is currently not included in the modeling of high-intensity lasermatter interactions. As a result complete spin polarization of the plasma will increase the total power radiated by the plasma by up to $15 \%$ compared to the emission from an equivalent unpolarized plasma; this could provide an observable signature of the spin polarization. Moreover, we would expect this to cause an increased rate of laser absorption.

Note that the effect of spin on $\eta$ and $\gamma$ through the spindependent $g(\eta)$ is smaller than on the total power radiated ( $<5 \%$ instead of $15 \%$, as numerically tested). Therefore, it was neglected in the calculation of the degree of spin polarization. 


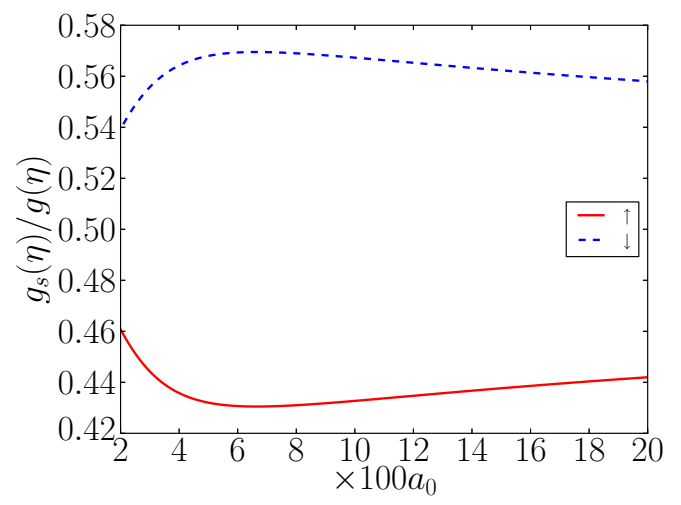

(a)

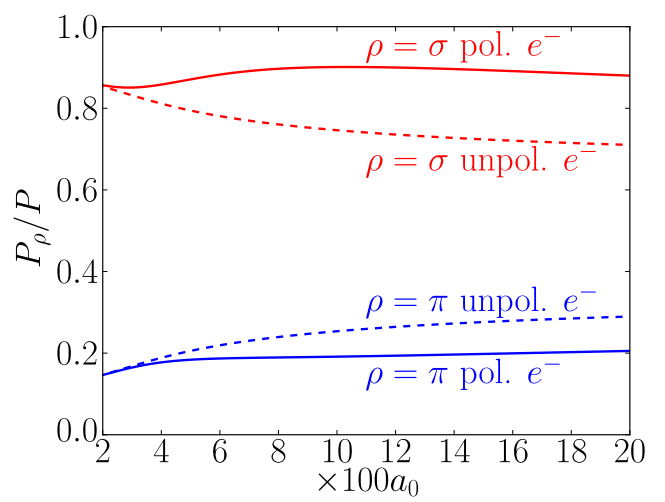

(b)

FIG. 2. (a) Spin-dependent $g^{s}(\eta)$ factor normalized to its unpolarized analog. (b) Power radiated by polarized and unpolarized electrons in both $\sigma$ and $\pi$ polarizations at $t=T$, normalized to the total power emitted by unpolarized electrons. All the quantities are plotted as functions of $a_{0}$.

The electron spin polarization also affects polarization of the emitted $\gamma$-ray photons. The power radiated in both $\sigma$ and $\pi$ polarizations, $P_{\sigma, \pi}=\sum_{s s^{\prime}} n^{s} \mathcal{P}_{\sigma, \pi}^{s s^{\prime}}$ at $t=T$, is plotted in Fig. 2(b), normalized to the total power emitted by unpolarized electrons. Electron spin polarization causes the relative power radiated as $\sigma$ photons to increase by up to $30 \%$ compared to the unpolarized case (and vice versa for $\pi$ photons). The relative yields of $\sigma$ and $\pi$ photons provide another signature of the spin polarization of the plasma.

The $\gamma$-ray photons emitted by the electrons can decay to pairs in the electromagnetic fields of the laser pulses by the multiphoton Breit-Wheeler process. Pair cascades become important rapidly as $a_{0}$ exceeds $1200\left(I=2 \times 10^{24} \mathrm{~W} / \mathrm{cm}^{2}\right)$ $[23,36-38]$. The polarization of the $\gamma$-ray photons has been shown to modify the rate of pairs produced by up to $30 \%$ [39]. The modification to the polarization of the $\gamma$-ray photons, caused by the spin polarization of the electrons, would be expected to produce a reduction in the rate of pair production of the same order, and thus, both electron and positron spin and photon polarization must be included in cascade simulations, yet they are currently neglected. The generated positrons will also spin polarize (parallel to $\zeta$ ). Although prolific pair production by a cascade will add source terms to equations for $d n^{\uparrow} / d \tau$ and $d n^{\downarrow} / d \tau$ (plus two additional equations for positrons), we would not expect a qualitative change to the spin-polarization trend in the magnetic node. Indeed, the rapid increase in the rate of pair production with laser intensity means that either the interaction is in a regime where a cascade does not occur and the number of positrons is small or the cascade is rapidly quenched as the number density of pairs reaches the relativistic critical density and the source term shuts off.

The spin magnetization of electrons in a plasma at the magnetic node, resulting from the spin polarization, can be deduced by multiplying Eq. (12) by $\mu_{B} n_{0}$ [40], where $\mu_{B}$ is the Bohr magneton. Assuming the plasma density is equal to the relativistic critical density (upper limit for laser propagation), the spin magnetization for $1 \mu \mathrm{m}$ wavelength lasers with $I>5 \times 10^{22} \mathrm{~W} / \mathrm{cm}^{2}\left(a_{0}>200\right)$ is $M \sim \mathrm{kG}$ after one laser period. This quantity may be considered another observable effect of spin polarization.

\section{CONCLUSIONS}

We have shown that electrons in a plasma created by two counterpropagating, ultraintense $\left(a_{0}>200\right.$; $I>5 \times 10^{22} \mathrm{~W} / \mathrm{cm}^{2}$ ) laser pulses can spin polarize (to $10 \%-70 \%$ ) on a femtosecond time scale. In this laser configuration, assuming the plasma is sufficiently rarefied that collective effects are negligible, the only experimental parameter that influences the degree of spin polarization is the laser intensity since the wavelength is currently fixed to $\sim 1 \mu \mathrm{m}$ by technological limitations.

Spin polarization can enhance the radiation reaction force on electrons and positrons in the plasma by up to $15 \%$ compared to the prediction for unpolarized electrons. Consequently, the power radiated by the plasma is enhanced by the same percentage. The polarization of the radiated $\gamma$-ray photons is also modified, by $30 \%$, potentially reducing the number of pairs produced in the plasma by $30 \%$.

The spin polarization must therefore be accounted for in the modeling of next-generation laser-matter interactions. The possibility of producing spin-polarized electrons and positrons with ultraintense lasers also opens up new applications. Polarized electrons are fundamental for the study of particle physics and are used in the spin-polarized electron spectroscopy.

The data required to reproduce the results in this paper are available from the University of York at https://doi. org/10.15124/1afd25a0-a1e3-49ec-afa8-2e5f6d868124.

\section{ACKNOWLEDGMENTS}

This work was funded by the UK Engineering and Physical Sciences Research Council (Grant No. EP/M018156/1), by the Science and Technology Facilities Council (Grant No. ST/G008248/1), and by the Knut and Alice Wallenberg Foundation. The authors are grateful to A. Di Piazza for useful and stimulating discussions. 
[1] G. Mourou, C. Labaune, M. Dunne, N. Naumova, and V. Tikhonchuk, Plasma Phys. Controlled Fusion 49, B667 (2007).

[2] A. Di Piazza, C. Müller, K. Hatsagortsyan, and C. Keitel, Rev. Mod. Phys. 84, 1177 (2012).

[3] P. Goldreich and W. H. Julian, Astrophys. J. 157, 869 (1969).

[4] R. D. Blandford and R. L. Znajek, Mon. Not. R. Astron. Soc. 179, 433 (1977).

[5] A. Macchi, M. Borghesi, and M. Passoni, Rev. Mod. Phys. 85, 751 (2013).

[6] R. Ekman, F. A. Asenjo, and J. Zamanian, Phys. Rev. E 96, 023207 (2017).

[7] T. Erber, Rev. Mod. Phys. 38, 626 (1966).

[8] V. I. Ritus, J. Sov. Laser Res. 6, 497 (1985).

[9] C. Ridgers, J. G. Kirk, R. Duclous, T. Blackburn, C. Brady, K. Bennett, T. Arber, and A. Bell, J. Comput. Phys. 260, 273 (2014).

[10] A. Di Piazza, K. Z. Hatsagortsyan, and C. H. Keitel, Phys. Rev. Lett. 105, 220403 (2010).

[11] D. Burke, R. Field, G. Horton-Smith, J. Spencer, D. Walz, S. Berridge, W. Bugg, K. Shmakov, A. Weidemann, C. Bula et al., Phys. Rev. Lett. 79, 1626 (1997).

[12] P. Zhang, C. Ridgers, and A. Thomas, New J. Phys. 17, 043051 (2015).

[13] D. Seipt, T. Heinzl, M. Marklund, and S. S. Bulanov, Phys. Rev. Lett. 118, 154803 (2017).

[14] T. Grismayer, M. Vranic, J. Martins, R. Fonseca, and L. Silva, Phys. Plasmas 23, 056706 (2016).

[15] E. N. Nerush, I. Y. Kostyukov, A. M. Fedotov, N. B. Narozhny, N. V. Elkina, and H. Ruhl, Phys. Rev. Lett. 106, 035001 (2011).

[16] L. Ji, A. Pukhov, E. Nerush, I. Y. Kostyukov, B. Shen, and K. Akli, Phys. Plasmas 21, 023109 (2014).

[17] D. D. Sorbo, D. R. Blackman, R. Capdessus, K. Small, C. SladeLowther, W. Luo, M. J. Duff, A. P. L. Robinson, P. McKenna, Z.-M. Sheng et al., arXiv:1706.04153.

[18] A. A. Sokolov and I. M. Ternov, Synchrotron Radiation (Akademie, Berlin, 1968).

[19] S. Belomesthnykh, A. Bondar, M. Yegorychev, V. Zhilitch, G. Kornyukhin, S. Nikitin, E. Saldin, A. Skrinsky, and G. Tumaikin, Nucl. Instrum. Methods Phys. Res., Sect. A 227, 173 (1984).
[20] A. Vauth and J. List, Int. J. Mod. Phy. Con. Ser. 40, 1660003 (2016).

[21] A. V. Subashiev, Yu. P. Yashin, J. E. Clendenin, and Yu. A. Mamaev, Phys. Low Dimens. Struct. 1, 1 (1998).

[22] H. Siegmann, J. Phys. Condens. Matter 4, 8395 (1992).

[23] A. R. Bell and J. G. Kirk, Phys. Rev. Lett. 101, 200403 (2008).

[24] B. King, Phys. Rev. A 91, 033415 (2015).

[25] V. Bargmann, L. Michel, and V. L. Telegdi, Phys. Rev. Lett. 2, 435 (1959).

[26] J. Vieira, C.-K. Huang, W. Mori, and L. Silva, Phys. Rev. Spec. Top. Accel. Beams 14, 071303 (2011).

[27] W. Heisenberg and H. Euler, Z. Phys. 98, 714 (1936).

[28] V. N. Baier and V. M. Katkov, Sov. Phys. JETP 26, 854 (1968).

[29] J. G. Kirk, A. Bell, and I. Arka, Plasma Phys. Controlled Fusion 51, 085008 (2009).

[30] V. A. Bordovitsyn, Synchrotron Radiation Theory and Its Development: In Memory of I. M. Ternov (1921-1996), edited by V. A. Bordovitsyn (World Scientific, Singapore, 1999).

[31] S. Mane, Y. M. Shatunov, and K. Yokoya, Rep. Prog. Phys. 68, 1997 (2005).

[32] U. I. Uggerhøj, Rev. Mod. Phys. 77, 1131 (2005).

[33] K. K. Andersen, J. Esberg, H. Knudsen, H. D. Thomsen, U. I. Uggerhøj, P. Sona, A. Mangiarotti, T. J. Ketel, A. Dizdar, and S. Ballestrero, Phys. Rev. D 86, 072001 (2012).

[34] V. B. Berestetskii, E. M. Lifshitz, and L. P. Pitaevskii, Quantum Electrodynamics (Pergamon Press, Oxford, 1982), Vol. 4.

[35] J. Kirk, Plasma Phys. Controlled Fusion 58, 085005 (2016).

[36] A. M. Fedotov, N. B. Narozhny, G. Mourou, and G. Korn, Phys. Rev. Lett. 105, 080402 (2010).

[37] S. S. Bulanov, C. B. Schroeder, E. Esarey, and W. P. Leemans, Phys. Rev. A 87, 062110 (2013).

[38] T. Grismayer, M. Vranic, J. L. Martins, R. A. Fonseca, and L. O. Silva, Phys. Rev. E 95, 023210 (2017).

[39] B. King, N. Elkina, and H. Ruhl, Phys. Rev. A 87, 042117 (2013).

[40] J. D. Jackson, Classical Electrodynamics (Wiley, New York, 1999). 